\title{
Early orthodontic treatment reduced incisal trauma in children with class II malocclusions
}

\author{
Abstracted from \\ Thiruvenkatachari B, Harrison JE, Worthington HV, O'Brien KD. \\ Orthodontic treatment for prominent upper front teeth (Class II malocclusion) in children. Cochrane Database Syst Rev 2013; 11: Art. \\ No.: CD003452. DOI: 10.1002/14651858.CD003452.pub3. \\ Address for correspondence: Luisa Fernandez Mauleffinch, Review Group Co-ordinator, Cochrane Oral Health Group, MANDEC, School \\ of Dentistry, University of Manchester, Higher Cambridge Street, Manchester, M15 6FH, UK. E-mail: luisa.fernandez@manchester.ac.uk
}

\section{Question: What is the best orthodontic treatment approach for prominent upper front teeth?}

Data sources The Cochrane Oral Health Group's Trials Register, the Cochrane Central Register of Controlled Trials (CENTRAL), Medline and Embase.

Study selection Randomised controlled trials (RCTs) of orthodontic treatments (either one- or two-phase) with any type of orthodontic braces (removable, fixed, functional) or head-braces compared with late treatment with any type of orthodontic braces or head-braces; or, on any type of orthodontic braces or head-braces compared with no treatment or another type of orthodontic brace or appliance to correct prominent upper front teeth.

Data extraction and synthesis Study selection, risk of bias assessment and data extraction were carried out independently by at least two reviewers. The primary measure of effect was over jet measured in millimetres or by any index of malocclusion. Odds ratios (ORs) and 95\% confidence intervals ( $\mathrm{Cls}$ ) were used for dichotomous outcomes, mean differences (MDs) and 95\% Cls for continuous outcomes and a fixed- effect model for meta-analyses as there were fewer than four studies.

Results Seventeen studies involving 791 patients were included. The overall quality of the evidence was low with only two of the 17 studies being assessed as at low risk of bias. Three trials $(n=343)$ compared early (two-phase) treatment (7-11 years of age) with a functional appliance with adolescent (one-phase) treatment.

Statistically significant differences in over jet, ANB and PAR scores were found in favour of functional appliance when the first phase of early treatment was compared with observation in the children due to receive treatment in adolescence. However, there was no evidence of a difference in the over jet between the groups at the end of treatment. A statistically significant reduction in the incidence of incisal trauma (OR $0.59,95 \% \mathrm{Cl} 0.35$ to $0.99, \mathrm{P}=0.04$ ) in favour of two-phase treatment with functional appliance was seen. The incidence of incisal trauma was clinically significant with $29 \%$ (54/185) of patients reporting new trauma incidence in the adolescent (one-phase) treatment group compared to only $20 \%$ (34/172) of patients receiving early (two-phase) treatment.

Two trials ( $\mathrm{n}=285$ ) compared early (two-phase) treatment using headgear, with adolescent (one-phase) treatment. Statistically significant differences in over jet and ANB were found in favour of headgear when the first phase of early treatment was compared with observation in the children due to receive treatment in adolescence. However, there was no evidence of a difference in the over jet between the groups at the end of treatment. The incidence of incisal trauma was, however, statistically significantly reduced in the two-phase treatment group, the adolescent treatment group having twice the incidence of incisal trauma $(47 / 120)$ compared to the young children group (27/ 117).

Two trials $(n=282)$ compared different types of appliances (headgear and functional appliance) for early (two-phase) treatment. At the end of the first phase of treatment statistically significant differences, in favour of functional appliances, were shown with respect to final over jet only. At the end of phase two, there was no evidence of a difference between appliances with regard to over jet, PAR score or the incidence of incisal trauma.

Late orthodontic treatment for adolescents with functional appliances showed a statistically significant reduction in over jet of $-5.22 \mathrm{~mm}(95 \% \mathrm{Cl}-6.51$ to $-3.93, \mathrm{P}<0.00001)$ and ANB of $-2.37^{\circ}$ ( $95 \% \mathrm{Cl}-3.01$ to $-1.74, \mathrm{P}<0.00001$ ) when compared to no treatment (very low quality evidence).

There was no evidence of a difference in over jet when Twin Block was compared to other appliances. However, a statistically significant reduction in $\mathrm{ANB}\left(-0.63^{\circ}, 95 \% \mathrm{Cl}-1.17\right.$ to $\left.-0.08, \mathrm{P}=0.02\right)$ was shown in favour of Twin Block. There was no evidence of a difference in any reported outcome when Twin Block was compared with modifications of Twin Block.

Conclusions The evidence suggests that providing early orthodontic treatment for children with prominent upper front teeth is more effective in reducing the incidence of incisal trauma than providing one course of orthodontic treatment when the child is in early adolescence. There appear to be no other advantages for providing treatment early when compared to treatment in adolescence.

\section{Commentary}

The semantic simplicity of the central issue in this Cochrane review in no way detracts from either the potential of outcomes or the gravity of the issue addressed. The controversy on early treatment, functional jaw orthopaedics, single-phase /two phase treatments has dominated orthodontic thought almost since the period of existence of the specialty.

Point and counterpoint will always coexist in the realm of 
critical thinking but yet the results of this review provide clinical directions and perhaps a transformation of the early orthodontics.

The central focus of the review is simple, the orthodontic management of prominent upper front teeth in children. Firstly, the treatment assessment relates to that performed exclusively by orthodontists, secondly, the best time for treatment, either in two phases from 7-11 years or in a single phase in adolescence from 11-16 years and thirdly, the different appliances used by orthodontists to achieve this. All these issues have been controversial and this review provides some crucial answers. That the review would have a significant impact on orthodontic thinking would be an understatement.

Given the implications of the outcomes, it becomes crucial to establish the validity of the review. The background put forward succinctly by the authors purports to the prominence of upper front teeth which affects a quarter of the children in the UK and would perhaps be a common denominator in a global perspective. The probability of injury and distress caused by the appearance mandates a clinical intervention.

So the first issue that needs answers is do you correct it when you see it albeit with two-phase therapy or do you treat with a single phase in adolescence? The issue has been addressed earlier and the 2007 Cochrane review by Harrison, O'Brien and Worthington summarises no distinct advantage from early treatment.

The search of the databases is comprehensive with no restrictions to language or publication dates preceding 17th April 2013. The Cochrane Oral Health Group's trial register, The Cochrane library, Medline and Embase would bring forth all the relevant studies. The complexity of the review is under scribed by the lack of uniform treatment strategies and appliances. The selection criteria detailed look at all randomised controlled trials on early treatment of children and adolescents below 16 years of age, either one-phase or twophase with removable, functional or fixed appliances.

The word head brace used by the authors would imply head gears, compared with late treatment with any type of appliance as also headgears, any type of interventions compared with no treatment at all or treatment with any other type of appliance to correct prominent upper front teeth.

The diversity of studies, interventions and outcomes does complicate the review and the authors have correctly observed that the quality of evidence is low.

The 17 trials included eventually yield data from 721 participants. By now the issue of prominent upper front teeth stands correlated to class 2 div 1 malocclusions where perhaps the effect of the appliances used would be as much to reduce the prominence of upper front teeth as to reposition the mandible with the eventual intent of a functional or growth modification change.

The strength of the review obviously comes from the stringency of processes with a screening of extracted results, independent assessment of risk bias, odds ratios and $95 \% \mathrm{CI}$ for dichotomous outcomes, mean differences and $95 \% \mathrm{CI}$ for continuous outcomes. The model for a meta-analysis is a fixed effect model as there are fewer than four studies. The flow diagram effectively summarises the issue of evidence related to orthodontics with a screening of 1572 records, assessment of 117 full text articles for eligibility with 17 studies being eventually included in a quantitative analysis. The assessment of risk of bias is impressive and adds to the strength of the review.
The great variability of outcomes measured in the diverse studies and the array of cephalometric analysis used would confound the outcomes being estimated from the included studies. The authors would need to be commended for finding a common skein of truth related to the objectives of the study and meta-analysis. The data analysis is comprehensive but direct. The estimation of mean difference, $95 \%$ CI leads to an effect size. The data analysis is detailed and covers all the parameters in the review.

The summary of the main results does provide interesting outcomes. In the issue of two-phase versus one-phase treatment for children between the ages of 7-11 years with prominent front teeth, there is a both a clinically and statistically significant improved incisor prominence irrespective of whether the child received treatment with a headgear or a functional appliance. There will be concomitant improvement in the relationship of the upper and lower jaws.

The issue of dental trauma in young children is resolved when compared to single-phase adolescent treatment, although the growth modifying effects may neither be clinically nor statistically significant. If one looks at treatment provided in one phase in early adolescence, the reduction in incisor prominence is significant but the change in the skeletal pattern may be miniscule and insignificant.

The primary outcome is the prominence of upper front teeth, which relates to the over jet measured in millimetres or by any index of malocclusion. The secondary outcomes encompass the relation of upper and lower jaws, the issues of self-esteem, patient satisfaction, TMJ issues and the number of visits to complete treatment. It is in this context that the scope of this review addresses a whole array of problems that are unresolved in clinical orthodontics.

There is a blurring of lines; are prominent upper teeth always related to Class 2 div 1 malocclusions? Three studies have been excluded, as they did not involve treatment of patients with Class 2 div 1 malocclusion.

In most of the studies incorporated in the review, were prominent upper teeth or the over jet the prime objective of treatment, or was the correction of the jaw relationship primary, and effects on upper incisors an additional outcome?

The subtle question also arises as to what lessons you take away from this Cochrane review when several previous ones have addressed some or most of the issues, perhaps in a disparate manner.

The serious reader of this review will find several answers that the previous reviews have not given. It is evident that the prime concern of orthodontists is and continues to be the issues related to the jaw relationships seen in Class 2 Div 1 malocclusions. A variety of appliances compared and used in the contributing trials are in one form or another functional appliances or headgears intended to modify growth.

So orthodontic thought still remains focused on the jaw relationships rather than the issue of prominent upper front teeth, injury or issues of self-esteem. Here the answers are clear, that these appliances have a miniscule role in modifying growth or significantly affecting the jaw relationships, but all of them do reduce procumbency of the incisors with the associated advantages of reduced injury and improved self-esteem.

The evidence also seems to point a finger to one-phase treatment in adolescence to manage both the growth as well as the occlusion. 
Do we need an introspection of orthodontic thinking, should early treatment focus more on reducing incisal trauma, poor self-esteem and all the related outcomes of incisor procumbency? There are more efficient methods of reducing prominent incisors with $2 \times 4$ fixed appliances. A realistic perception of growth modification and timing of treatment is critical.

This review could be one of the significant scientific efforts at evidence-based modification of clinical practice. For the astute practitioner and orthodontist there is a clear message:

- Early treatment for prominent upper front teeth in children reduces trauma as well as poor self-esteem and social adjustment. It is important to correct it when you see it.

- The choice of the appliance will rest with the clinician; there is limited evidence to support growth modification or two-phase therapy.

The authors need to be complimented for this overarching review that has so much to contribute to treatment decisions and clinical practice. The results go well beyond the simplicity of the primary issue of prominent front teeth in children.

Anmol S Kalha

I.T.S. Dental College, Hospital and Research Center, Greater Noida, NCR, India

Evidence-Based Dentistry (2014), 15, 18-20. doi: 10.1038/sj.ebd.6400986 